\title{
Inhibition of mTOR signaling protects photoreceptor cells against serum deprivation by reducing oxidative stress and inducing $\mathrm{G}_{\mathbf{2}} / \mathrm{M}$ cell cycle arrest
}

\author{
BIN FAN $^{1}$, FU-QAING LI ${ }^{1}$, JING-YAO SONG ${ }^{1}, \mathrm{XU} \mathrm{CHEN}^{2}$ and GUANG-YU LI ${ }^{1}$ \\ ${ }^{1}$ Department of Ophthalmology, Second Hospital of Jilin University, Changchun, Jilin 130041; \\ ${ }^{2}$ Department of Ophthalmology, Zhongshan Hospital of Fudan University, Shanghai 200032, P.R. China
}

Received November 5, 2015; Accepted February 17, 2016

DOI: $10.3892 / \mathrm{mmr} .2016 .5011$

\begin{abstract}
The mammalian target of rapamycin (mTOR) pathway is a crucial cellular signaling hub, which integrates internal and external cues to modulate the cell cycle, protein synthesis and metabolism. The present study hypothesized that inhibiting mTOR signaling may induce cells to enter lower and more stable bioenergetic states, in which neurons have greater resistance to various insults. Neurotrophin withdrawal from photoreceptor cells (661W cells) was mimicked using serum deprivation, and the neuroprotective mechanisms were studied following suppression of the mTOR pathway. Treatment with an mTOR specific inhibitor, rapamycin, reduced intracellular levels of reactive oxygen species, suppressed oxidative stress, and attenuated mitochondrial dysfunction. In addition, inhibiting mTOR signaling induced a $\mathrm{G}_{2} / \mathrm{M}$ cell cycle arrest, thus providing an opportunity to repair damaged DNA and block the cell death cascade. These results suggested that inhibition of mTOR had a neuroprotective effect on serum-deprived $661 \mathrm{~W}$ cells. In conclusion, the mTOR pathway is a critical molecular signal for cell cycle regulation and energy metabolism, and inhibiting the mTOR pathway may attenuate neurotrophin withdrawal-induced damage. These observations may provide evidence for the treatment of retinal degenerative disease, since inducing neurons into a lower and more stable bioenergetic state by blocking mTOR signaling may slow the progression of neurodegenerative diseases.
\end{abstract}

\section{Introduction}

The mammalian target of rapamycin (mTOR) is a large protein kinase of the phosphatidylinositol 3-kinase (PI3K)-related

Correspondence to: Professor Guang-Yu Li, Department of Ophthalmology, Second Hospital of Jilin University, 4026 Yatai Street, Changchun, Jilin 130041, P.R. China

E-mail: liguangyu@aliyun.com

Key words: $\mathrm{mTOR}, \mathrm{G}_{2} / \mathrm{M}$ cell cycle arrest, rapamycin, photoreceptor cells, oxidative stress kinase family (1). There are two conserved TOR cell complexes: TOR complex (TORC)1 and TORC2 (2). Upstream and downstream effectors of TOR have been identified using cultured mammalian cells. Upstream, multiple signals exist, including insulin signaling through PI3K and AKT, and energy signaling [via the adenosine triphosphate:adenosine monophosphate (AMP) ratio] through AMP-activated protein kinase. These signals converge in the tuberous sclerosis (TSC)1-TSC2 complex, which serves as a GTPase exchange factor for Rheb, whereas Rheb in GTP-bound form activates mTOR through direct binding (3). Downstream of mTOR, S6 kinase 1 (S6K1) and eukaryotic initiation factor 4E-binding protein (4EBP1) are the two most studied effectors, which are phosphorylated by mTORC1 but not mTORC2. Phosphorylation of these two effectors, particularly S6K1 phosphorylation at threonine 389, has been widely used to indicate mTORC1 activity. These effectors regulate translational initiation and control protein synthesis (4).

The present study hypothesized that inhibiting mTOR signaling may induce cellular stability, in which energy consumption and cellular metabolism decrease. In this state, damaged nuclear DNA is primarily repaired and mitochondrial dysfunction is controlled. Subsequently, the cell death cascade is suppressed. Therefore, mTOR inhibition-induced cell stability may be considered promising protection against neurodegenerative insults.

Neurotrophins exert diverse effects in the development and regeneration of neural circuits in the vertebrate visual system (5). In the retina, neurotrophins influence proliferation, neurite outgrowth and cell survival in the visual system in vitro and in vivo (6). Neurotrophin availability is critical for controlling normal cell death, since the majority of retinal neurons depend on growth factors for their survival, and cells may die when they lack adequate survival factors (6). In addition, neurotrophins rescue photoreceptors from degeneration (7). The present study used serum deprivation to mimic neurotrophin loss in retinal neurons, and explored the neuroprotective mechanisms following suppression of the mTOR pathway.

The $661 \mathrm{~W}$ cell line was cloned from the retinal tumors of a transgenic mouse line, and expresses simian virus 40T antigen under the control of the human interphotoreceptor 
retinol-binding protein promoter. These cells usually grow as a monolayer and behave as photoreceptor cells, which express blue and green cone pigments, transducin and cone arrestin, but not retinal pigment epithelial cell-specific proteins. Furthermore, 661W cells are sensitive to photooxidative stress, similar to normal retinal photoreceptor cells (8).

The present study used the $661 \mathrm{~W}$ cell line to investigate the molecular mechanisms underlying serum deprivation-induced cell death. In addition, the mTOR pathway was blocked using a specific inhibitor, rapamycin. The results demonstrated that inhibiting mTOR resulted in increased stability of photoreceptor cells and cell cycle arrest at $\mathrm{G}_{2} / \mathrm{M}$ stage. Furthermore, intracellular levels of reactive oxygen species (ROS) and apoptotic markers were markedly decreased. Therefore, inhibiting the mTOR pathway may have a neuroprotective effect against serum deprivation-induced cell death.

\section{Materials and methods}

Chemicals and reagents. Cell culture media and additives were purchased from Hyclone (GE Healthcare Life Sciences, Logan, UT, USA). Plastic cultureware was obtained from Greiner Bio-One GmbH (Frickenhausen, Germany). Rabbit antibodies against phosphorylated (p)-P70S6 kinase (P70S6K) (cat. no. 11284), p-4EBP1 (cat. no. 11223) and mouse $\beta$-actin (cat. no. 21800-1) were purchased from Signalway Antibody LLC (College Park, MD, USA). Rabbit antibodies against p-mTOR (cat. no. BS4706), heme oxygenase-1 (HO-1) (cat. no. BS6626), cyclin D1 (cat. no. BS6532) and cyclin D3 (cat. no. BS6139) were purchased from Bioworld Technology, Inc. (St. Louis Park, MO, USA). Rabbit antibodies against poly (ADP-ribose) polymerase 1 (PARP-1) (cat. no. 9542), cleaved caspase-3 (cat. no. 9662) and cyclin D2 (cat. no. 3741) were purchased from Cell Signaling Technology, Inc. (Danvers, MA, USA). Goat anti-apoptosis inducing factor (AIF) (cat.no. sc-9416) was obtained from Santa Cruz Biotechnology, Inc. (Dallas, TX, USA). Rapamycin, dichloro-dihydro-fluorescein diacetate (DCFH-DA), JC-1, MitoTracker Green and other reagents were purchased from Sigma-Aldrich Shanghai Trading Co., Ltd. (Shanghai, China).

Cell culture. The 661W photoreceptor cell line was generously provided by Dr. Muayyad Al-Ubaidi (Department of Cell Biology, University of Oklahoma Health Sciences Center, Oklahoma City, OK, USA). Cells were cultured in Dulbecco's modified Eagle's medium supplemented with $10 \%$ heat-inactivated fetal calf serum (Hyclone; GE Healthcare Life Sciences) and $1 \%$ penicillin/streptomycin, at $37^{\circ} \mathrm{C}$ in a humidified atmosphere containing $5 \% \mathrm{CO}_{2}$. Cells have a doubling time of $\sim 20 \mathrm{~h}$ under these conditions, and were passaged by trypsinization at a ratio of 1:6 every 3-4 days. For the serum deprivation experiments, the $661 \mathrm{~W}$ cells were cultured in 96- or 24-well plates for $24 \mathrm{~h}$ with normal medium, washed with PBS three times and then cultured with serum-free medium for 1, 2, 4 or 6 days. For the rapamycin experiments, the $661 \mathrm{~W}$ cells were additionally treated with $100 \mathrm{nM}$ rapamycin during serum deprivation for 2,4 or 6 days.

Intracellular ROS measurement. Intracellular ROS were measured using the oxidation-sensitive fluorescent probe
DCFH-DA (9). Cells were cultured in 6-well plates for 2 days, were washed twice with fresh medium, and were then incubated with $10 \mu \mathrm{M}$ DCFH-DA at $37^{\circ} \mathrm{C}$ for $20 \mathrm{~min}$. Oxidized 2,7-dichlorofluorescein fluorescence was visualized under the IX-ULWCD fluorescent microscope (Olympus Corporation, Tokyo, Japan). Fluorescent intensities were measured using ImageJ software, version 1.46 (National Institutes of Health, Bethesda, MD, USA). Relative fluorescence intensities of the cells were assessed using the following formula (10): Biomarker relative intensity $=[$ Foreground intensity (cell staining) / surface area] / [background intensity / surface area].

Propidium iodide (PI) staining. The $661 \mathrm{~W}$ cells were cultured in 6-well plates for $24 \mathrm{~h}$. After serum deprivation at the indicated time points, cells were stained with PI solution $(2 \mu \mathrm{g} / \mathrm{ml})$ and incubated in the dark for $10 \mathrm{~min}$ at room temperature. PI-positive cells were visualized under the IX-ULWCD fluorescent microscope (Olympus Corporation). For quantitative analysis, cells were harvested by trypsinization, rinsed with phosphate-buffered saline (PBS), and analyzed by flow cytometry (FACSCanto II; BD Biosciences, San Diego, CA, USA).

Cell cycle analysis. Cell cycle distribution was analyzed by flow cytometry (11). Six groups of cells $\left(\sim 1 \times 10^{6}\right.$ cells/group) were harvested by trypsinization, rinsed with PBS and fixed with cold $70 \%$ ethanol at $4^{\circ} \mathrm{C}$ overnight. Cells were then washed twice with PBS and re-suspended in $1 \mathrm{ml}$ staining solution $(50 \mu \mathrm{g} / \mathrm{ml}$ PI, $50 \mu \mathrm{g} / \mathrm{ml}$ RNaseA, $0.1 \%$ Triton X-100 in citrate buffer; $\mathrm{pH} 7.8$ ) at room temperature for $30 \mathrm{~min}$. Cells were acquired and the percentage of cells in each cell cycle stage was analyzed by flow cytometry (FACSCanto II; BD Biosciences).

Mitochondrial-membrane potential assay. JC-1 selectively accumulates within intact mitochondria to form multimeric J-aggregates, which emit fluorescent light at $590 \mathrm{~nm}$ (red) at a higher membrane potential (12). In the present study, the cells were seeded in 6-well plates, and cultured with serum-free medium for 2 days. Subsequently, the medium was removed, and the cells were washed with $\mathrm{Ca}^{2+} / \mathrm{Mg}^{2+}$-free PBS. Cells were stained with $\mathrm{JC}-1(10 \mu \mathrm{g} / \mathrm{ml})$ for $30 \mathrm{~min}$ at $37^{\circ} \mathrm{C}$ and were examined under the IX-ULWCD fluorescent microscope (Olympus Corporation) at $590 \mathrm{~nm}$.

Mitochondrial staining. Mitochondria of the $661 \mathrm{~W}$ cells were stained using MitoTracker Green, as described previously by Stojkovic et al(13). Briefly, 661W cells were incubated in serum-free medium containing $0.2 \mathrm{mM}$ MitoTracker Green for $10 \mathrm{~min}$ at $37^{\circ} \mathrm{C}$. Subsequently, the $661 \mathrm{~W}$ cells were washed several times in fresh medium, mounted on glass slides, and were observed under the IX-ULWCD fluorescent microscope (Olympus Corporation) at $490 \mathrm{~nm}$. Images were captured and analyzed using ImageJ software, version 1.46 (National Institutes of Health).

Western blotting. Immunoblot assay was performed as described previously by Li et al(14). The $661 \mathrm{~W}$ cells were sonicated in protein lysis buffer, and a bicinchoninic acid assay was used to determine protein concentration. An equal amount of cell lysate $(20 \mu \mathrm{g})$ was dissolved in sample buffer, and samples 
were boiled for $3 \mathrm{~min}$. Electrophoresis was performed using $10 \%$ polyacrylamide gels containing $0.1 \%$ sodium dodecyl sulfate. Proteins were transferred to nitrocellulose membranes and were subsequently blocked for $1 \mathrm{~h}$ at $25^{\circ} \mathrm{C}$ in $5 \%(\mathrm{w} / \mathrm{v})$ non-fat dried milk. The blots were incubated for $3 \mathrm{~h}$ at room temperature with primary antibodies $(1: 1,000)$, followed by an incubation with goat anti-rabbit, rabbit anti-mouse or anti-goat biotinylated secondary antibodies (1:1,000; Sigma-Aldrich; cat. nos. A0545, A9044 and A5420, respectively) for $1.5 \mathrm{~h}$. Signals were developed using enhanced chemiluminescence and images were captured using a charge-coupled device camera (Tanon Science \& Technology Co., Ltd., Shanghai, China). Densitometry analysis was performed using Quantity One software, version V4.62 (Bio-Rad Laboratories, Inc., Hercules, CA, USA).

Statistical analysis. Each experiment was repeated at least three times. Data are presented as the mean \pm standard error of the mean. Differences between the means were evaluated using one-way analysis of variance followed by a Bonferroni test with the SPSS software, version 17 (SPSS Inc., Chicago, IL, USA). Significance for all cases was $\mathrm{P}<0.001$.

\section{Results}

Inhibition of mTOR protects $661 \mathrm{~W}$ cells from serum deprivation-induced injury. The present study investigated the effects of serum deprivation on $661 \mathrm{~W}$ cells by measuring cell death using PI. Compared with the cells cultured in normal medium (Fig. 1A), on day 3 of serum deprivation photoreceptor cells underwent programmed cell death, and were positively stained for PI (Fig. 1B). Conversely, rapamycin (100 nM) attenuated serum starvation-induced cell death, as evidenced by fewer PI-positive cells (Fig. 1C). Cell death was monitored with flow cytometry up to 6 days, by which time nearly all cells had died. As shown in Fig. 1D, cell death in the serum-starved group increased over time compared with in the control cells cultured in normal medium. On day 3, serum deprivation killed $\sim 50 \%$ of the cells, and by day $480 \%$ of the cells were dead. By days 5 and $6,>90 \%$ of the cells were dead, as measured by PI staining and flow cytometry. The percentage of dead control cells at this time point was $\sim 15 \%$.

Subsequently, the effects of mTOR inhibition on serum-deprived photoreceptor cells were assessed. Initially, it was confirmed that rapamycin inhibited mTOR signaling by western blot analysis. As shown in Fig. 1E, rapamycin (100 nM) markedly suppressed the expression levels of downstream factors of mTOR, as evidenced by reduced protein expression of p-P70S6 and p-4EBP1 on days 2 and 4 of serum deprivation. Consistent with inhibition of mTOR, cell death analysis indicated that rapamycin exerted neuroprotective effects against serum deprivation in photoreceptor cells, with cell death maintained at $<20 \%$ up to 6 days (Fig. 1D; $\mathrm{P}<0.001$ vs. the serum-deprived group). These results suggest that mTOR inhibition may protect photoreceptor cells from neurotrophin withdrawal.

Inhibition of mTOR suppresses serum starvation-induced oxidative stress. As shown in Fig. 2A-C, intracellular ROS increased after 2 days of serum deprivation, as compared with in the normally cultured cells, as measured using the oxidation-sensitive fluorescent probe DCFH-DA, which fluoresces green under fluorescent microscopy. Quantitative analysis indicated that cellular ROS were significantly upregulated in serum-starved cells compared with normally cultured cells $(\mathrm{P}<0.001)$. Conversely, treatment with rapamycin suppressed cellular ROS generation in serum-deprived cells, and reduced DCFH-DA fluorescence intensity was detected (Fig. 2D; $\mathrm{P}<0.001$ vs. the serum-deprived group). To evaluate oxidative stress in $661 \mathrm{~W}$ cells, the expression of $\mathrm{HO}-1$, an enzyme modulating antioxidant defense, was detected. As shown in Fig. 2E serum deprivation upregulated HO-1 expression from day 1 , whereas the addition of rapamycin markedly attenuated serum starvation-induced increases in HO-1. These results indicate that inhibiting the mTOR pathway may suppress serum starvation-induced oxidative stress in $661 \mathrm{~W}$ cells.

Inhibition of mTOR helps attenuate mitochondrial dysfunction. Mitochondrial membrane potential was analyzed using JC-1 staining. The majority of $661 \mathrm{~W}$ cells were stained fluorescent red, indicating the presence of healthy mitochondria (Fig. 2F), whereas serum-deprived 661W cells exhibited less fluorescence (Fig. 2G). Treatment with rapamycin restored mitochondrial potential in serum-deprived cells, as evidenced by increased red fluorescence (Fig. 2H). Mitochondrial swelling denotes mitochondrial dysfunction, due to opening of the mitochondrial permeability transition pore. Mitochondrial morphology was assessed using the mitochondrial-specific MitoTracker Green. As shown in Fig. 2I-K, mitochondria in normal-cultured cells exhibited clear dot-shaped structures (Fig. 2I), whereas the serum-starved cells exhibited increased swelling with ambiguous boundaries (Fig. 2J). As predicted, treatment with rapamycin improved mitochondrial morphology in serum-deprived cells, and they were able to maintain a clear dot shape (Fig. $2 \mathrm{~K}$ ). These results indicate that inhibition of mTOR may contribute to restoration of mitochondrial function, improving mitochondrial membrane potential and mitochondrial structure.

Inhibition of mTOR leads to $G_{2} / M$ cell cycle arrest. Cell cycle checkpoints and DNA repair systems closely cooperate to maintain genomic integrity of cells damaged by external or internal insults, including intracellular ROS (15). To investigate the neuroprotective mechanisms underlying the effects of mTOR suppression, cell cycle progression was investigated in photoreceptor cells. The $661 \mathrm{~W}$ cells were treated with rapamycin $(100 \mathrm{nM})$ under serum-deprived condition, and on days 2 and 4 cell cycle distribution was assessed. As shown in Fig. 3A-D, rapamycin significantly increased the number of serum-deprived cells in $\mathrm{G}_{2} / \mathrm{M}$ phase; cells accumulated from 28.5 to $38.2 \%$ on day 2 (Fig. 3C), and from 45.1 to $54.5 \%$ by day 4 (Fig. 3D; $\mathrm{P}<0.001$ vs. the serum-deprived group). Notably, serum deprivation as an external insult resulted in significant cell cycle arrest at $\mathrm{G}_{2} / \mathrm{M}$ phase, compared with in the normally cultured cells $(\mathrm{P}<0.001)$. Subsequently, the effects of mTOR inhibition on the protein expression levels of cyclins (key cell cycle regulatory molecules) were analyzed by western blotting. Rapamycin markedly reduced cyclin D2 and D3 protein expression levels on days 2 and 4 under 


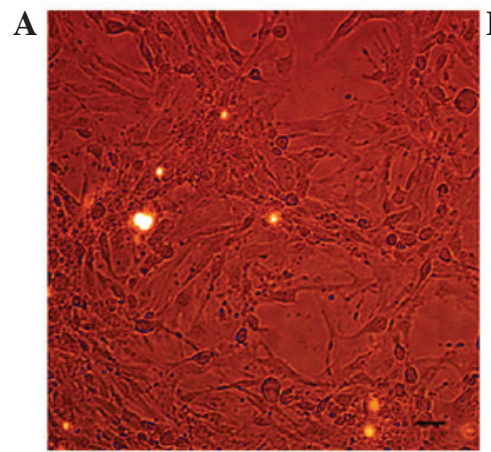

D

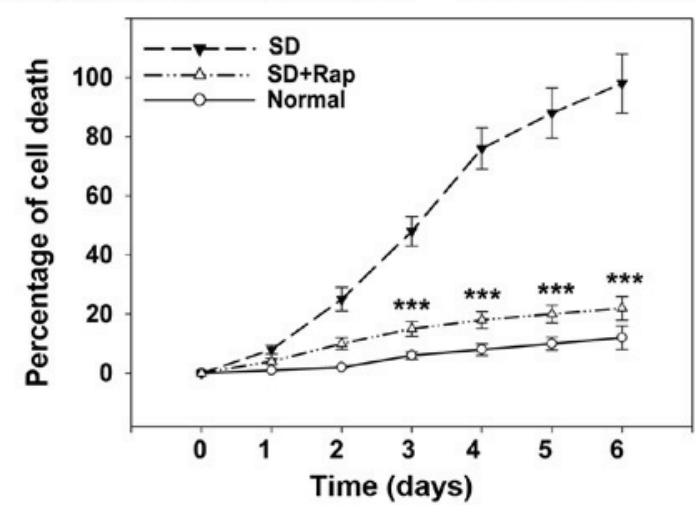

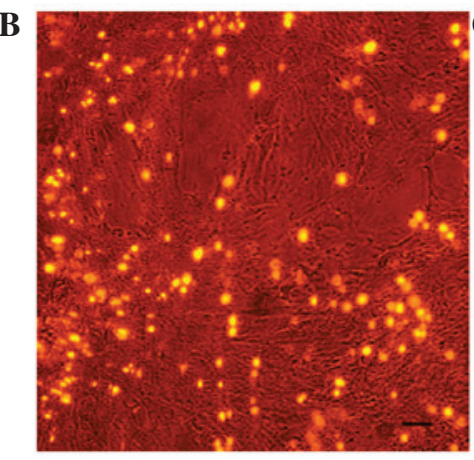

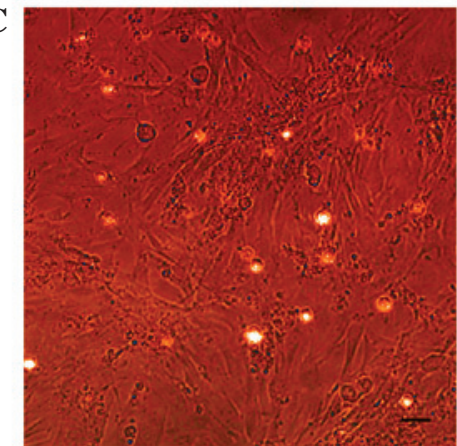

$\mathbf{E}$

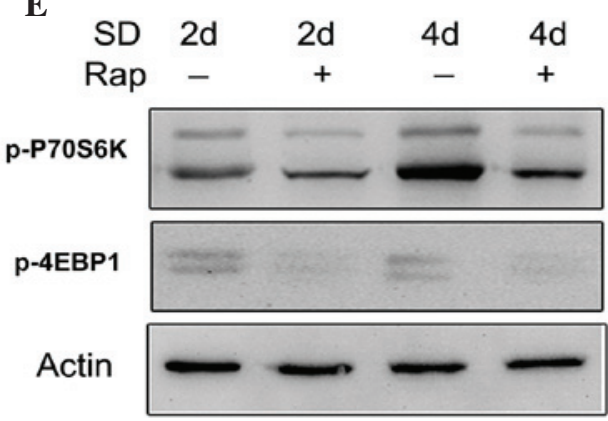

Figure 1. Inhibition of mammalian target of rapamycin signaling attenuates serum deprivation-induced cell death. (A-C) Cell death was evaluated using propidium iodide (PI) staining (scale bars $=100 \mu \mathrm{m}$ ). (A) $661 \mathrm{~W}$ control cells were cultured under normal conditions. (B) 661W cells were cultured in serum-free medium for 3 days. (C) $661 \mathrm{~W}$ cells were treated with $100 \mathrm{nM}$ rapamycin alongside serum deprivation for 3 days. Scale bar, $100 \mu \mathrm{m}$. (D) Quantification of cell death via PI staining. ${ }^{* * *} \mathrm{P}<0.001$ vs. the serum-deprived group. Assays were performed in quadruplicate, and data are presented as the mean \pm standard error of the mean. Normal, cells cultured in normal medium; SD, cells cultured in serum-free medium; SD+Rap, cells cultured in serum-free medium and treated with $100 \mathrm{nM}$ rapamycin. (E) Treatment with rapamycin (100 nM) reduced phosphorylated (p)-P70S6 kinase (P70S6K) and p-eukaryotic initiation factor 4E-binding protein (4EBP1) expression on days 2 and 4 of serum deprivation.

serum-deprived conditions, whereas cyclin D1 expression was markedly increased following treatment with rapamycin for 2 and 4 days (Fig. 3E). These results suggest that mTOR inhibition may lead to $\mathrm{G}_{2} / \mathrm{M}$ cell cycle arrest in serum-deprived $661 \mathrm{~W}$ cells, which may be due to the modulation of cyclin protein expression.

Inhibition of mTOR reduces expression of apoptotic markers. As shown in Fig. 4A, serum deprivation increased the expression of cleaved caspase- 3 from day 2 to 6 , as evidenced by detection of a $19 \mathrm{kDa}$ protein band. Quantitative analysis revealed that the relative amount of cleaved caspase- 3 in the serum-deprived cell lysate was significantly higher than in the normally cultured cell lysate $(\mathrm{P}<0.001)$. As a substrate of caspase-3, PARP-1 is normally cleaved into two protein forms while the caspase-dependent pathway is activated. Western blot analysis confirmed that concentration of cleaved PARP-1 increased in the serum-deprived cells compared with the control cells $(\mathrm{P}<0.001)$. To illustrate serum deprivation-induced cell death, a caspase-independent pathway marker, AIF, was measured. The $57 \mathrm{kDa}$ active form of AIF was upregulated under serum deprivation conditions from days 2 to 6 , and was significantly higher than in normally cultured cells $(\mathrm{P}<0.001)$. Western blotting confirmed that rapamycin significantly attenuated upregulation of cleaved caspase- 3 and PARP-1 at days 2 and 4 and the active form of AIF was reduced (Fig. 4B; $\mathrm{P}<0.001$ vs. the serum-deprived group). These results suggest that the caspase-dependent and -independent pathways were activated by serum starvation and mTOR inhibition may block apoptotic pathways by suppressing the expression of apoptotic factors.

\section{Discussion}

mTOR is an evolutionarily conserved serine/threonine kinase, which responds to numerous stimuli, including growth factors, nutrients, energy status and oxygen, to regulate cellular proliferation and growth (16). The present study demonstrated that serum deprivation, as an external insult, mimics neurotrophin withdrawal and causes photoreceptor cell death. Conversely, mTOR inhibition was neuroprotective, and was able to rescue $661 \mathrm{~W}$ cells from serum starvation.

Mitochondria are chief producers of ROS via the electron transport chain. A decline in mitochondrial function, including damaged membrane potential, leads to enhanced ROS production (17). Once ROS production is increased, due to changes in oxidative mitochondrial metabolism, damage to cellular components and cell death may occur (18). The results of the present study are consistent with those of a previous study, and indicated that serum deprivation led to damaged mitochondrial membrane potential and increased ROS production (19). After 2 days of serum deprivation, the majority of $661 \mathrm{~W}$ cells exhibited reduced JC-1 red fluorescence, indicating the presence of impaired mitochondria. However, abnormal mitochondrial activities were inhibited by treatment with rapamycin. Consistently, increased ROS production was detected in 
$\mathbf{A}$
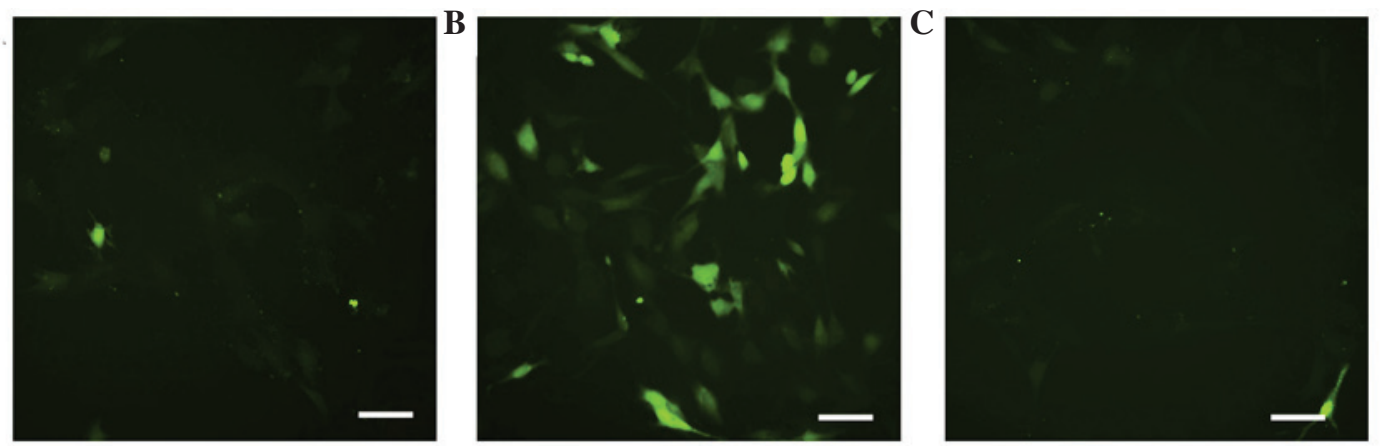

D
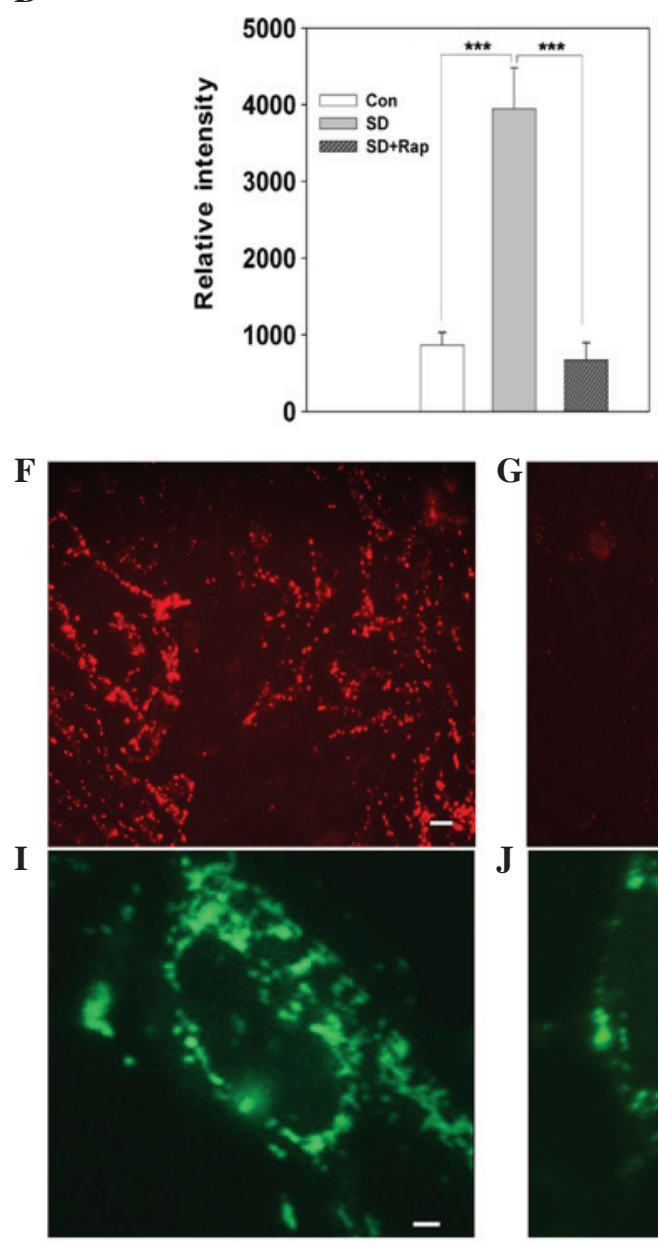

I

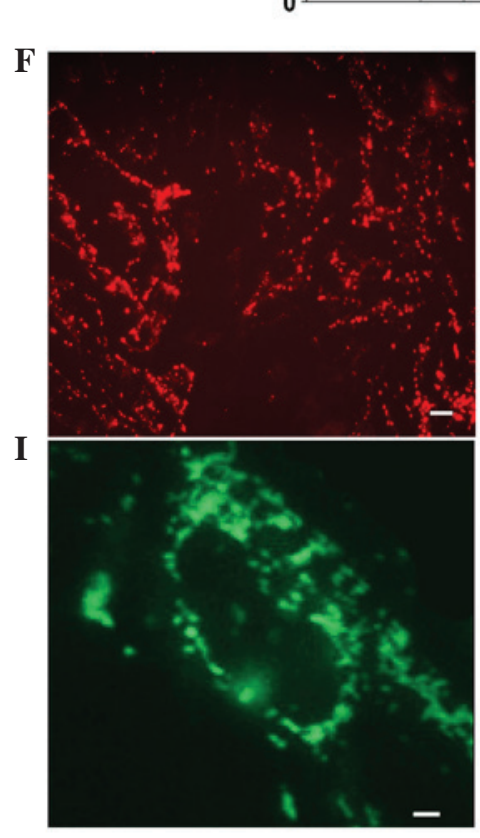

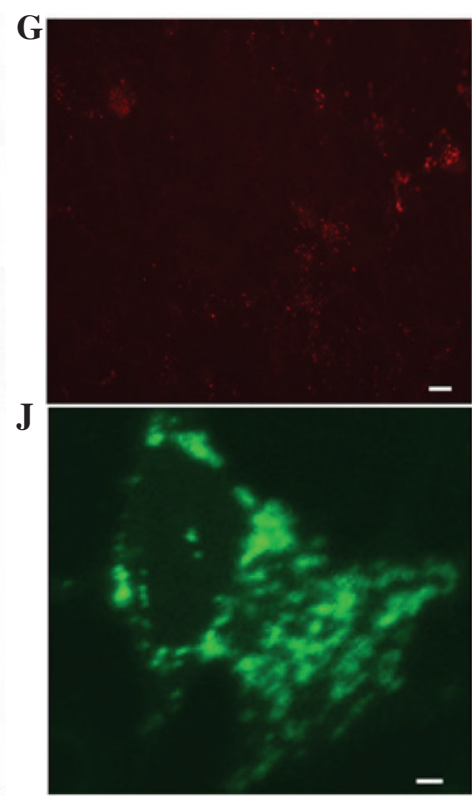

$\mathbf{E}$
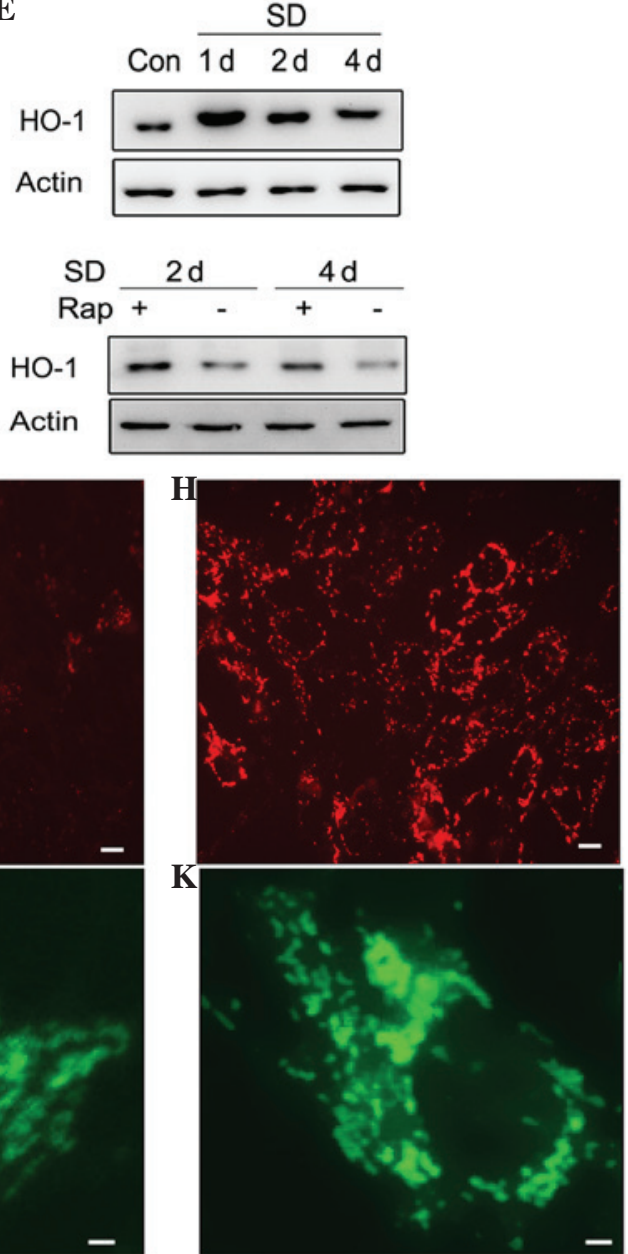

Figure 2. Inhibition of mammalian target of rapamycin signaling suppresses serum starvation-induced oxidative stress and improves mitochondrial function. (A-C) Intracellular reactive oxygen species (ROS) were measured using dichloro-dihydro-fluorescein diacetate (DCFH-DA). (A) 661W control cells were cultured in normal medium for 2 days. (B) $661 \mathrm{~W}$ cells were cultured in serum-free medium for 2 days. (C) $661 \mathrm{~W}$ cells were cultured in serum-free medium and were treated with $100 \mathrm{nM}$ rapamycin for 2 days. (D) Fluorescent intensities were measured and relative fluorescence was compared. Data were obtained from at least three independent experiments and are presented as the mean \pm standard error of the mean. ${ }^{* * * *} \mathrm{P}<0.001$. Con, cells cultured in normal medium; SD, cells cultured in serum-free medium; SD+Rap, cells cultured in serum-free medium and treated with $100 \mathrm{nM}$ rapamycin. (E) Cells were harvested and lysed for immunoblot detection of heme oxygenase-1 (HO-1). SD, serum deprivation; Rap, $100 \mathrm{nM}$ rapamycin. (F-H) Mitochondrial membrane potential was assessed by JC-1 staining under a fluorescent microscope. (F) 661W cells were cultured in normal medium for 2 days. (G) $661 \mathrm{~W}$ cells were cultured in serum-free medium for 2 days. (H) $661 \mathrm{~W}$ cells were cultured in serum-free medium and were treated with $100 \mathrm{nM}$ rapamycin for 2 days. Scale bar, $50 \mu \mathrm{m}$. (I-K) Mitochondria in $661 \mathrm{~W}$ cells were stained with MitoTracker Green. (I) $661 \mathrm{~W}$ cells were cultured with normal medium for 2 days. (J) $661 \mathrm{~W}$ cells were cultured with serum-free medium for 2 days. (K) $661 \mathrm{~W}$ cells were cultured in serum-free medium and were treated with $100 \mathrm{nM}$ rapamycin for 2 days. Scale bar, $10 \mu \mathrm{m}$.

serum-starved cells, whereas rapamycin decreased cellular ROS levels. Notably, mitochondrial structure appeared swollen in response to serum deprivation, whereas following rapamycin treatment mitochondria exhibited a clear dot pattern, according to the results of MitoTracker Green staining. Mitochondria are essential to multicellular life, therefore apoptotic pathways target this organelle in various ways; the apoptotic stimuli may cause mitochondrial swelling via membrane pore formation, or they may increase mitochondrial membrane permeability, allowing leakage of apoptotic effectors (13).

Cell growth and proliferation are major cellular functions controlled by mTORC1 (20), which is reported to preferentially 

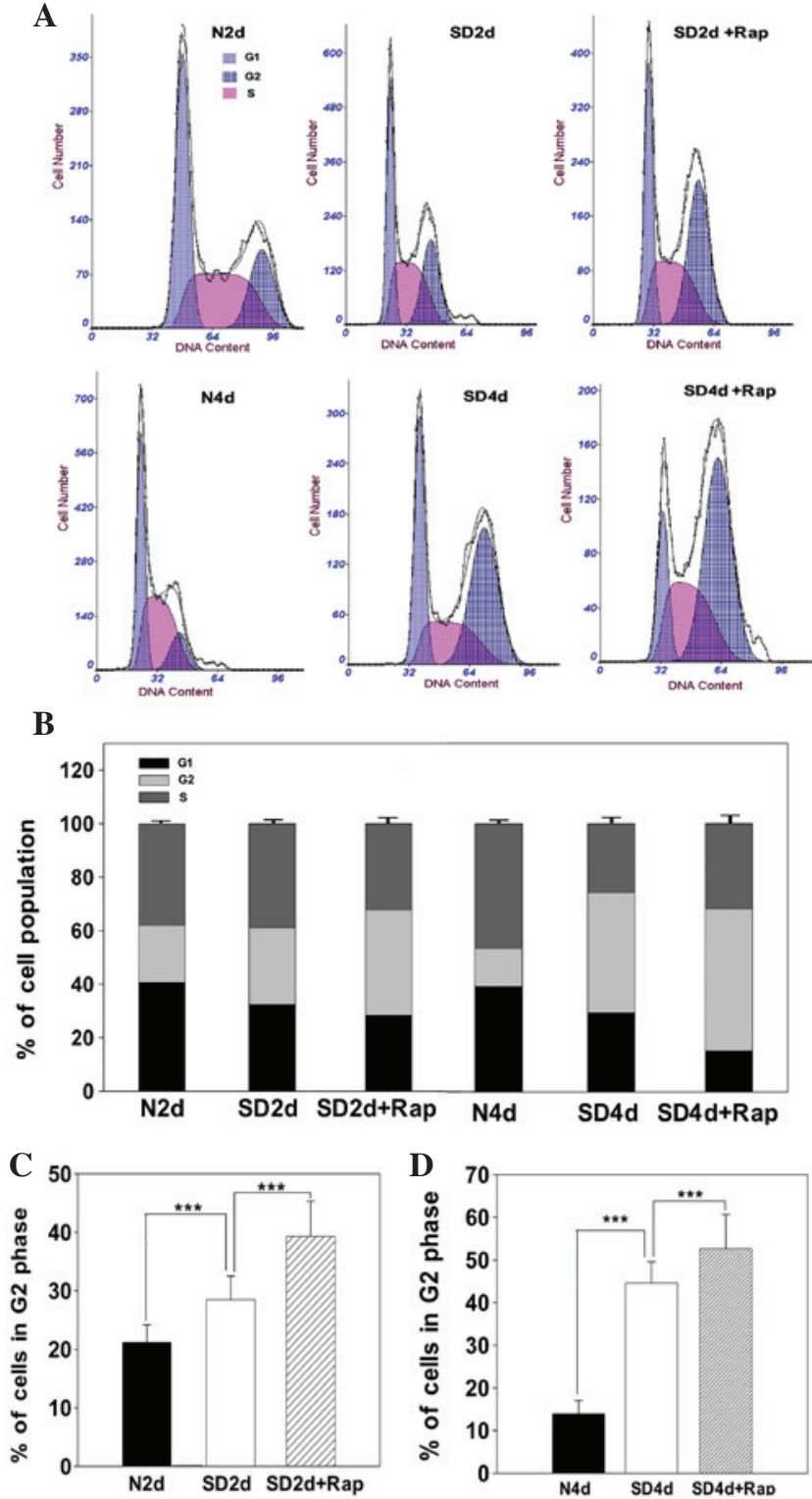

$\mathbf{E}$

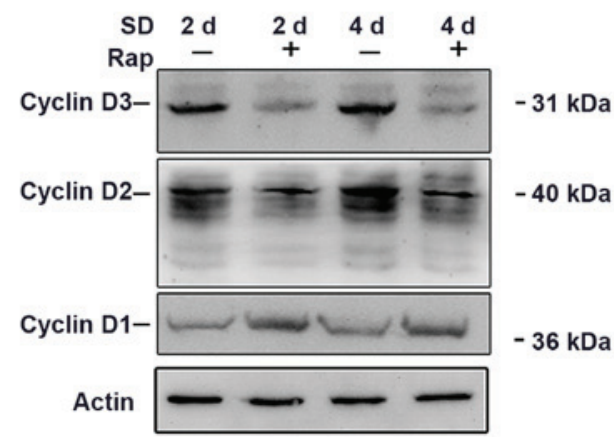

Figure 3. Inhibition of mammalian target of rapamycin signaling leads to $\mathrm{G}_{2} / \mathrm{M}$ cell cycle arrest. (A) Cell cycle distribution was measured with flow cytometry and propidium iodide staining. (B) Percentage of cells in each cell cycle phase. Data are presented as the mean \pm standard error of the mean (SEM) of three independent experiments at each concentration. (C and D) Percentage of $\mathrm{G}_{2}$ phase distribution in the presence/absence of $100 \mathrm{nM}$ rapamycin at the indicated time. Data are presented as the mean \pm SEM of three independent experiments. ${ }^{* * *} \mathrm{P}<0.001$. N2d, normal medium for 2 days; SD2d, serum deprivation for 2 days; SD2d+Rap, rapamycin with serum deprivation for 2 days; N4d, normal medium for 4 days; SD4d, serum deprivation for 4 days; SD4d+Rap, rapamycin with serum deprivation for 4 days. (E) Rapamycin $(100 \mathrm{nM})$ reduced cyclin D3 and D2 expression, but increased cyclin D1 expression on days 2 and 4. Each experiment was repeated at least 3 times, and a representative blot is shown.
A
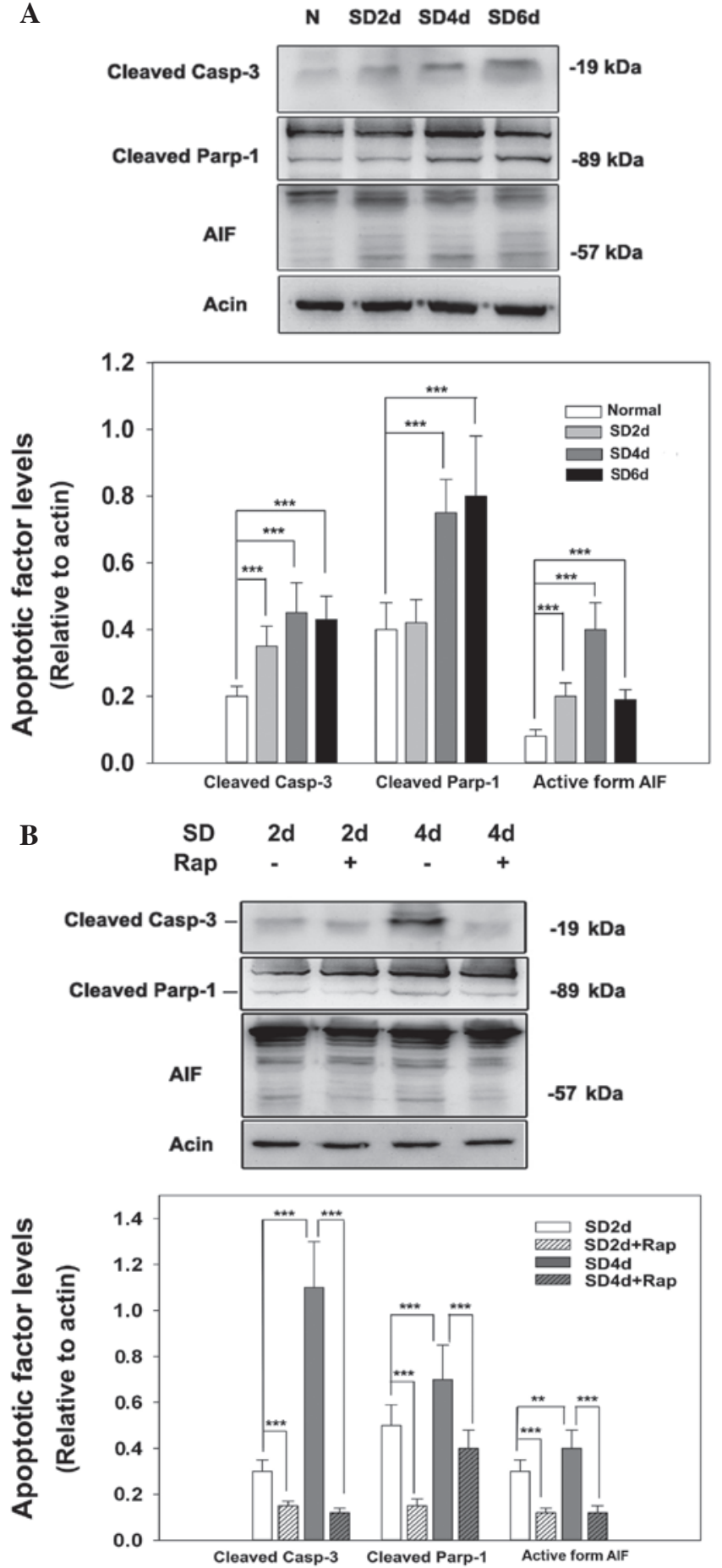

Figure 4. Inhibition of mammalian target of rapamycin reduces the expression of apoptotic markers. (A) $661 \mathrm{~W}$ cells were cultured with serum-free medium for the designated times. Cells were then harvested and lysed for immunoblot analysis. N, normal medium; SD2d, serum deprivation for 2 days; SD4d, serum deprivation for 4 days; SD6d, serum deprivation for 6 days. (B) $661 \mathrm{~W}$ cells were cultured with/without rapamycin while serum-deprived for the designated times. Cells were then harvested and lysed for immunoblot analysis. SD, serum deprivation; Rap, $100 \mathrm{nM}$ rapamycin. Each experiment was repeated at least 3 times, and a representative blot is presented. Data were obtained from at least three independent experiments and are presented as the mean \pm standard error of the mean. ${ }^{* *} \mathrm{P}<0.05,{ }^{* * * *} \mathrm{P}<0.001$. Casp-3, caspase-3; Parp-1, poly (ADP-ribose) polymerase 1; AIF, apoptosis inducing factor.

drive cell growth through S6K1 and cell proliferation through 4EBP1 (21). The cell cycle consists of four phases: $G_{1}$ phase, $\mathrm{S}$ (or DNA synthesis) phase, $\mathrm{G}_{2}$ phase, and $\mathrm{M}$ (or mitosis) 
phase. The results of the present study demonstrated that inhibition of the mTOR pathway induced $661 \mathrm{~W}$ cell cycle arrest at $\mathrm{G}_{2} / \mathrm{M}$ stage, as evidenced by flow cytometry. This cell cycle arrest occurred in cells cultured under serum-deprived conditions. The $\mathrm{G}_{2}$ checkpoint prevents cells from entering mitosis when DNA is damaged, providing an opportunity for repair and cessation of damaged cell proliferation. DNA repair systems and cell cycle checkpoints closely cooperate to maintain genomic integrity of cells damaged by external or internal insults, including intracellular ROS and ionizing radiation. Cells at $G_{1} / S$ and $S$ phases prevent replication of damaged DNA, and those at $\mathrm{G}_{2} / \mathrm{M}$ phase prevent segregation of modified chromosomes. Cells that lack, or have altered, genes involved in DNA double-strand break repair and cell cycle checkpoints die early and are genomically unstable (15). Eukaryotes possess various types of cyclins, all of which exhibit specialized functions. In animal cells, D-type cyclins are fundamental for re-entry into the cell cycle in response to extracellular signals (22). The results of the present study indicated that inhibition of mTOR signaling caused $661 \mathrm{~W}$ cell cycle arrest at $\mathrm{G}_{2} / \mathrm{M}$ phase and upregulated cyclin D1 expression; however, cyclin D2 and cyclin D3 expression was downregulated. Cyclin D1 is critical for regulating $G_{1} / S$-phase transition. When cells are arrested in $\mathrm{G}_{2} / \mathrm{M}$ phase, cyclin D1 is likely increased as a compensatory response. In addition, cyclin D1 governs DNA damage repair by recruiting DNA repair complexes. When nuclear DNA is damaged by excessive intracellular ROS during oxidative stress, cyclin D1 may be increased, in order to repair damaged DNA. The observation that mTOR inhibition caused a decrease in cyclin D2 and D3 expression was consistent with the results of a previous study, thus indicating their importance in regulating $\mathrm{G}_{2} / \mathrm{M}$ arrest (23).

Apoptosis is a well-orchestrated type of cell death, which is responsible for maintenance of normal tissue homeostasis and removal of damaged, old or infected cells. Caspase-dependent apoptosis is finely regulated by a series of pro-apoptotic proteins, including caspase-3 and PARP-1, which are markers of apoptotic pathways (24). Increased active caspase- 3 and cleaved PARP-1 are products of the activated caspase-dependent pathway. Decreased cleaved caspase-3 and PARP-1 expression was detected in the rapamycin-treated $661 \mathrm{~W}$ cells in the present study, thus suggesting that rapamycin may prevent retinal neurons from subsequent pro-apoptotic insults. In addition, a typical caspase-independent pathway was investigated. AIF is a flavoprotein, which is confined to the mitochondrial intermembrane space in healthy cells. Upon lethal signaling, AIF translocates, via the cytosol, to the nucleus where it binds to DNA and provokes caspase-independent chromatin condensation (25). The present study revealed that serum deprivation resulted in upregulation of the active form of AIF (57 kDa), thus suggesting that the caspase-independent pathway may be involved in $661 \mathrm{~W}$ cell death. Conversely, treatment with rapamycin reduced the expression of $57 \mathrm{kDa} \mathrm{AIF}$. Therefore, a proposed neuroprotective mechanism underlying the effects of mTOR inhibition may be suppression of caspase-dependent and -independent apoptotic pathways.

In conclusion, mTOR inhibition exerts protective effects against serum deprivation in $661 \mathrm{~W}$ cells, and induces a stable $\mathrm{G}_{2} / \mathrm{M}$ cell cycle arrest, in which energy consumption and cellular metabolism are largely decreased. In this state, damaged nuclear DNA is primarily repaired, mitochondrial dysfunction is restored, and the cell death cascade is suppressed, which may contribute to its anti-apoptotic effects. These findings suggested that rapamycin may be a potential therapeutic agent for the prevention of retinal degenerative diseases and neurotrophin defects. Inducing neurons into a lower and more stable bioenergetic state by blocking the mTOR pathway may be considered a therapeutic strategy to slow the progression of neurodegenerative diseases.

\section{Acknowledgements}

The present study was supported by grants from the National Natural Science Foundation of China (grant nos. 81100660, 30801271 and 81570864) and the Norman Bethune Program of Jilin University (grant no. 2012208).

\section{References}

1. Wullschleger S, Loewith R and Hall MN: TOR signaling in growth and metabolism. Cell 124: 471-484, 2006.

2. Loewith R, Jacinto E, Wullschleger S, Lorberg A, Crespo JL, Bonenfant D, Oppliger W, Jenoe P and Hall MN: Two TOR complexes, only one of which is rapamycin sensitive, have distinct roles in cell growth control. Mol Cell 10: 457-468, 2002.

3. Manning BD and Cantley LC: Rheb fills a GAP between TSC and TOR. Trends Biochem Sci 28: 573-576, 2003.

4. Hay N and Sonenberg N: Upstream and downstream of mTOR. Genes Dev 18: 1926-1945, 2004.

5. Harada T, Harada C and Parada LF: Molecular regulation of visual system development: More than meets the eye. Genes Dev 21: 367-378, 2007.

6. von Bartheld CS: Neurotrophins in the developing and regenerating visual system. Histol Histopathol 13: 437-459, 1998.

7. Wen R, Tao W, Li Y and Sieving PA: CNTF and retina. Prog Retin Eye Res 31: 136-151, 2012.

8. Tan E, Ding XQ, Saadi A, Agarwal N, Naash MI and Al-Ubaidi MR: Expression of cone-photoreceptor-specific antigens in a cell line derived from retinal tumors in transgenic mice. Invest Ophthalmol Vis Sci 45: 764-768, 2004.

9. Brem R, Li F, Montaner B, Reelfs O and Karran P: DNA breakage and cell cycle checkpoint abrogation induced by a therapeutic thiopurine and UVA radiation. Oncogene 29: 3953-3963, 2010.

10. Riethdorf S, Müller V, Zhang L, Rau T, Loibl S, Komor M, Roller M, Huober J, Fehm T, Schrader I, et al: Detection and HER 2 expression of circulating tumor cells: Prospective monitoring in breast cancer patients treated in the neoadjuvant GeparQuattro trial. Clin Cancer Res 16: 2634-2645, 2010.

11. Ma GF, Chen SY, Sun ZR, Miao Q, Liu YM, Zeng XQ, Luo TC, Ma LL, Lian JJ and Song DL: FoxP3 inhibits proliferation and induces apoptosis of gastric cancer cells by activating the apoptotic signaling pathway. Biochem Biophys Res Commun 430: 804-809, 2013.

12. Qi Y, Li Y, Zhang Y, Zhang L, Wang Z, Zhang X, Gui L and Huang J: IFI6 inhibits apoptosis via mitochondrial-dependent pathway in Dengue virus 2 infected vascular endothelial cells. PLoS One 10: e0132743, 2015.

13. Stojkovic M, Machado SA, Stojkovic P, Zakhartchenko V, Hutzler P, Gonçalves PB and Wolf E: Mitochondrial distribution and adenosine triphosphate content of bovine oocytes before and after in vitro maturation: Correlation with morphological criteria and developmental capacity after in vitro fertilization and culture. Biol Reprod 64: 904-909, 2001.

14. Li GY, Fan B and Jiao YY: Rapamycin attenuates visible light-induced injury in retinal photoreceptor cells via inhibiting endoplasmic reticulum stress. Brain Res 1563: 1-12, 2014.

15. Belli M, Sapora O and Tabocchini MA: Molecular targets in cellular response to ionizing radiation and implications in space radiation protection. J Radiat Res 43 (Suppl): S13-S19, 2002. 
16. Morita M, Gravel SP, Hulea L, Larsson O, Pollak M, St-Pierre J and Topisirovic I: mTOR coordinates protein synthesis, mitochondrial activity and proliferation. Cell Cycle 14: 473-480, 2015.

17. Du H, Duanmu M, Witte D and Grabowski GA: Targeted disruption of the mouse lysosomal acid lipase gene: Long-term survival with massive cholesteryl ester and triglyceride storage. Hum Mol Genet 7: 1347-1354, 1998.

18. Hamanaka RB and Chandel NS: Mitochondrial reactive oxygen species regulate cellular signaling and dictate biological outcomes. Trends Biochem Sci 35: 505-513, 2010.

19. Yan C, Ding X, Dasgupta N, Wu L and Du H: Gene profile of myeloid-derived suppressive cells from the bone marrow of lysosomal acid lipase knock-out mice. PLoS One 7: e30701, 2012.

20. Zhang H, Stallock JP, Ng JC, Reinhard C and Neufeld TP: Regulation of cellular growth by the Drosophila target of rapamycin dTOR. Genes Dev 14: 2712-2724, 2000.
21. Dowling RJ, Topisirovic I, Alain T, Bidinosti M, Fonseca BD, Petroulakis E, Wang X, Larsson O, Selvaraj A, Liu Y, et al: mTORC1-mediated cell proliferation, but not cell growth, controlled by the 4E-BPs. Science 328: 1172-1176, 2010.

22. Sherr CJ: G1 phase progression: Cycling on cue. Cell 79: 551-555, 1994.

23. Weidner C, Rousseau M, Plauth A, Wowro SJ, Fischer C, Abdel-Aziz H and Sauer S: Melissa officinalis extract induces apoptosis and inhibits proliferation in colon cancer cells through formation of reactive oxygen species. Phytomedicine 22 : 262-270, 2015

24. Siddiqui A, Hanson I and Andersen JK: Mao-B elevation decreases parkin's ability to efficiently clear damaged mitochondria: Protective effects of rapamycin. Free Radic Res 46: 1011-1018, 2012.

25. Polster BM: AIF, reactive oxygen species, and neurodegeneration: A 'complex' problem. Neurochem Int 62: 695-702, 2013. 